Evaluar.

2003, $n^{\circ} 3$ (julio)
Laboratorio de Evaluación Psicológica y Educativa. Facultad de Psicología Universidad Nacional de Córdoba (Argentina).ISSN N ${ }^{\circ}$ 1667-4545

\title{
Modelo Social Cognitivo del Desarrollo de Carrera
}

\author{
Olaz. F.* \\ *Lab. de Evaluación Psicológica y Educativa. Facultad de Psicología. Universidad Nacional de Córdoba.
}

\begin{abstract}
Resumen El objetivo del presente trabajo es el de revisar un modelo de gran importancia heurística, capacidad predictiva y explicativa del desarrollo de carrera. El Modelo de Lent, Brown \& Hackett (1994) se destaca como una propuesta de integración y unificación teórica entre diferentes constructos así como por su énfasis en la investigación empírica de las hipótesis propuestas por los autores. El modelo es un intento de extensión de la línea iniciada por Hackett \& Betz (1981), incorporando otros aspectos de la teoría Social Cognitiva y permite un análisis más complejo y refinado del comportamiento vocacional, ampliando los aportes que el constructo de la Autoeficacia ha podido realizar por sí solo.
\end{abstract}

Palabras Clave: Autoeficacia - Desarrollo Vocacional - Intereses Vocacionales - Elección vocacional - Expectativas de Resultados-

\section{Introducción}

A partir de la introducción del marco teórico Social Cognitivo (Bandura, 1987) dentro del campo de la Orientación Vocacional, han sido propuestos numerosos modelos explicativos del desarrollo vocacional.

La elaboración de modelos explicativos de las relaciones entre los constructos frecuentemente utilizados para brindar claridad al proceso de desarrollo del comportamiento vocacional, que incorporan numerosas variables social cognitivas, están permitiendo una mayor comprensión de este proceso. Modelos basados ya no solamente en consideraciones teóricas sino en los resultados de numerosas investigaciones empíricas permiten análisis más refinados dentro de marcos teóricamente más abarcativos que integran y unifican constructos aparentemente diversos.

Los modelos explicativos se inscriben dentro de dos grandes ramas: La teoría del aprendizaje social de la toma de decisiones vocacionales de Krumboltz (1996) y la 
teoría de la Autoeficacia aplicada al estudio del comportamiento vocacional de Hackett y Betz (1981) (Lent, Brown \& Hackett, 1994). Debido a la importancia heurística del modelo, su capacidad explicativa y predictiva así como la extensa investigación que se esta realizando dentro de este campo de estudio, el presente trabajo tiene como objetivo revisar el modelo de Lent, Brown \& Hackett (1994) para la explicación del desarrollo de carrera.

\section{EI Desarrollo de carrera desde la Teoría Social Cognitiva}

Schunk (1989) propuso uno de los primeros modelos explicativos del proceso de desarrollo de la autoeficacia en el ámbito educativo. El modelo postula una influencia recíproca entre la autoeficacia, variables de compromiso con las tareas y las conductas de logro. Si bien el modelo no ha sido desarrollado específicamente para la explicación del comportamiento vocacional, el autor señala que puede ser aplicado en diferentes áreas, incluyendo a este último. El modelo es representado a continuación.

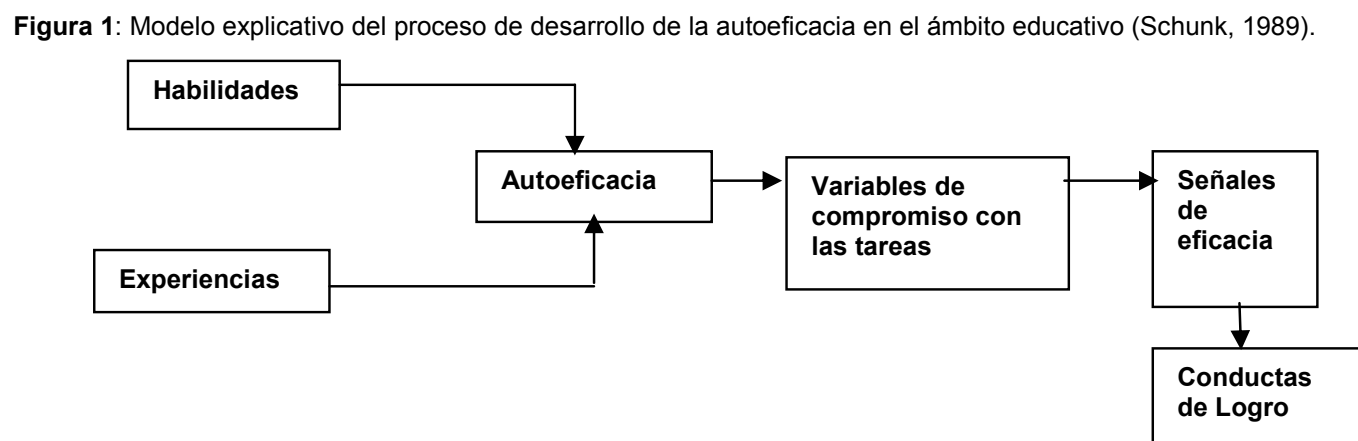

Al comienzo de una actividad educativa, los estudiantes difieren entre sí en las creencias que poseen acerca de su eficacia personal para adquirir conocimientos, desempeñar habilidades, y manejarse con el material de estudio. Esta variabilidad se debe a las experiencias educativas diferenciales de los sujetos así como a diferencias en características personales tales como habilidades y actitudes. Diferentes variables contextuales, de instrucción y otras variables asociadas al contexto de aprendizaje afectan a los sujetos mientras estos están comprometidos con las tareas académicas, brindándoles diferentes señales de eficacia personal. Los sujetos utilizan estas señales para evaluar su eficacia en actividades futuras. Estas señales incluyen los logros de 
rendimiento, los patrones de éxito y fracaso, las atribuciones, las comparaciones sociales, la credibilidad del persuasor, el establecimiento de metas y su consecución, las contingencias de refuerzo y las señales fisiológicas. A partir de estas señales, el sujeto elaborara juicios de autoeficacia que van a afectar la motivación y el rendimiento posterior en las tareas. A partir de la aparición de estos primeros modelos explicativos se multiplicaron los aportes teóricos y empíricos que fueron brindando abordajes analíticos más complejos y refinados que estos primeros modelos. La elaboración de estos modelos de mayor complejidad estará basada ya no solamente en consideraciones teóricas sino en los resultados de numerosas investigaciones empíricas e irán incorporando un creciente número de variables.

Se destaca principalmente el modelo propuesto por Lent, Brown \& Hackett (1994), un intento de extensión del trabajo iniciado por Hackett \& Betz (1981). El modelo propuesto por estos autores incorpora numerosas variables de la teoría Social Cognitiva para explicar en forma más compleja el comportamiento vocacional, representando así, un esfuerzo de integración y unificación teórica entre constructos aparentemente diversos, ampliando los aportes que el constructo de la Autoeficacia puede realizar por sí solo. El modelo se destaca además por su énfasis en la investigación empírica de las hipótesis propuestas por el modelo.

Lent, Brown \& Hackett (1994), utilizaron poderosos métodos estadísticos para la elaboración de su modelo y para la comprobación de las hipótesis de el derivadas. Uno de estos métodos es el análisis de ruta (Path Analysis) una extensión del modelo de regresión, técnica sumamente utilizada en la investigación destinada a comprobar hipótesis causales sin manipular las variables independientes. El análisis de ruta permite poner a prueba proposiciones teóricas acerca de la relación causal entre variables utilizando un sistema de ecuaciones lineales. Como señala Kerlinger \& Lee (2002) este procedimiento aún funciona como una herramienta de investigación útil para el desarrollo de un modelo conceptual que pueda ser comprobado de manera empírica. El procedimiento consiste en la construcción de un diagrama de ruta (input path diagram), que es una representación del modelo causal teorizado. Luego se procede al cálculo de un coeficiente, el coeficiente de ruta (path coeficient), que es un coeficiente de regresión estandarizado (Beta) y que muestra el efecto directo de las variables independientes sobre una variable dependiente del modelo hipotetizado. En 
algunos casos, cuando se compara la capacidad explicativa de diferentes modelos causales p. ej., se procede luego a un análisis estadístico denominado modelo de ecuaciones estructurales (Structural equation modeling, SEM), que permite determinar cual de los modelos teóricos es congruente con los datos obtenidos.

Otro procedimiento utilizado por estos autores para evaluar la capacidad predictiva del modelo es el Meta-análisis. El Meta-análisis es un procedimiento metodológico que emplea técnicas de medición y análisis estadístico sobre un conjunto de resultados de diversos estudios empíricos a los fines de integrar sus hallazgos con propósitos de generalización (Gómez Benito, 1987). Este procedimiento permite comprobar estadísticamente la medida en la cual una serie de estudios, tomados colectivamente, soportan o desconfirman hipótesis teóricas particulares.

El modelo de Lent, Brown \& Hackett (1994) enfatiza tres componentes: a) creencias de autoeficacia, b) expectativas de resultado y c) metas. El modelo incorpora a su vez otras variables personales (tales como aptitudes, género y etnicidad) así como variables contextuales, intentando explicitar la forma en la cual estos diferentes elementos se interrelacionan y la forma en la cual afectan a los intereses vocacionales, la elección de carrera y el posterior rendimiento.

Las expectativas de resultados han sido definidas como las creencias personales acerca de los posibles resultados de nuestras respuestas, y constituyen otro importante constructo de la teoría social cognitiva. Así como la autoeficacia se relaciona con los juicios acerca de nuestras capacidades personales de respuesta ( $i$ realmente puedo hacer esto?) las expectativas de resultado se refieren a las consecuencias imaginadas de llevarse a cabo determinadas conductas (si hago esto, ¿qué pasaría?) (Lent, Brown \& Hackett ,1994). Bandura (1987) distingue entre diferentes clases de expectativas de resultados tales como la anticipación de resultados físicos (dinero p. ej.), sociales (aprobación p. ej.), y autoevaluativos (autosatisfacción p. ej.) los cuales afectan de forma importante al comportamiento vocacional. En este marco teórico, el concepto de valores, muy utilizado en el campo de la Orientación Vocacional, es incorporado dentro del constructo expectativas de resultado ya que se considera que los intereses en una determinada actividad dependen, en parte, de los resultados anticipados de la participación en la actividad, junto con el valor relativo o la importancia otorgada por el individuo a estos resultados. Las expectativas de resultados se relacionan 
fuertemente con las creencias de autoeficacia precisamente porque estas creencias determinan en gran parte tales expectativas. De esta forma aquellas personas que se consideran capaces en una actividad particular anticiparan resultados exitosos. Sin embargo, la relación entre autoeficacia y expectativas de resultados no siempre es consistente. Un estudiante puede tener claro que el poseer habilidades para las matemáticas es esencial para un buen desempeño en una carrera relacionada, tal como Ingeniería, lo cual a su vez le asegurará un satisfactorio nivel de vida, pero si posee unas creencias débiles acerca de sus habilidades matemáticas van evitar elegir carreras y cursos relacionados. Unas creencias fuertes de autoeficacia y la anticipación de resultados también es posible. Un claro ejemplo sería el de un sujeto con fuertes creencias de autoeficacia para las matemáticas que anticipa bajas notas en un examen debido a que el profesor de la materia no ha aprobado a ninguno de sus compañeros de similar nivel de habilidad.

En la actualidad, Bandura $(1987,1997)$ ha realizado una clara distinción entre diferentes formas de expectativas de resultado y ha especificado las condiciones bajo las cuales éstos son totalmente determinados, parcialmente determinados, o no son determinados por las creencias de autoeficacia, así como las condiciones socioestructurales determinantes del grado de relación entre ambas variables. Algunas de estas condiciones en las cuales las creencias de autoeficacia y las expectativas de resultados no están directamente relacionados pueden ser aquellas situaciones en las cuales los resultados anticipados no son totalmente dependientes del rendimiento del sujeto (el alumno que anticipa malas notas no obstante su nivel de habilidad sería un buen ejemplo), o aquellas situaciones en las cuales los resultados son totalmente independientes del rendimiento de los sujetos, situaciones comunes en sistemas sociales rígidos y altamente estructurados.

Los diferentes roles representados por ambos constructos continúa siendo un área en estudio, pero numerosas investigaciones soportan las consideraciones de la teoría social cognitiva acerca del mayor poder predictivo de las creencias de autoeficacia (Pajares, 1997).

Otro importante constructo de la teoría social cognitiva son las metas, las cuales han sido definidas como la determinación de involucrarse en determinada actividad o de conseguir un determinado resultado en el futuro (Bandura,1987). El importante 
papel de las metas ha sido destacado en otras investigaciones (Lent, López, \& Bieschke,1991; Lent et al, 1993). Las metas ejercen su cualidad motivadora a través de la capacidad del sujeto de representar simbólicamente resultados deseados y de reaccionar autoevaluativamente basándose en criterios internos de rendimiento, haciendo depender la autosatisfacción al logro de éstos.

La teoría social cognitiva postula importantes relaciones de reciprocidad entre la autoeficacia, las expectativas de resultados y el sistema de metas (Bandura, 1987). Con frecuencia los investigadores no tienen en cuenta las complejas interacciones entre estas variables, concentrándose exclusivamente en el papel desempeñado por la autoeficacia, simplificando de esta forma los posibles interjuegos y la conducta consecuente, dejando muchas veces la impresión de que una autoeficacia alta es buena y una baja autoeficacia es mala. Esta sobresimplificación teórica no considera que la autoeficacia puede ser beneficiosa en algunas situaciones pero contraproducente en otras, dependiendo sus efectos de la relación compleja que esta mantiene con las expectativas de resultados, las aptitudes y conocimientos del sujeto, las metas y otras variables contextuales (Pajares, 1997).

Lent, Brown \& Hackett (1994) representan el desarrollo de intereses vocacionales, el proceso de elección de carrera y el rendimiento posterior en tres modelos diferentes pero interrelacionados. Los autores proponen una serie de relaciones teóricas así como ciertas vías causales, respetando el modelo de causalidad triádica al considerar que: a) a lo largo del desarrollo, los principales elementos teóricos ( persona, conducta y contexto) se van a influir unos con otros recíprocamente y b) en determinados momentos (o en determinadas personas) ciertas variables van a tener un predominio causal sobre otras.

\section{EI Modelo de Desarrollo de Intereses Vocacionales}

Los intereses vocacionales son definidos como patrones de gustos, aversiones, e indiferencias con respecto a actividades y ocupaciones relacionadas a una carrera. De acuerdo con Lent et al. (1994), el proceso de desarrollo vocacional incluye un modelo recíproco en el cual la autoeficacia y las expectativas de resultados influyen sobre los intereses. (vía 1 y 2). Es probable que las personas posean intereses perdurables en actividades en las cuales se consideren a sí mismos eficaces y en las cuales anticipen 
resultados positivos. Los intereses determinan a su vez las intenciones y metas (vía 3) que se fijará el sujeto, las cuales determinaran a su vez la elección de determinadas actividades y su práctica posterior (vía 4). El resultado de esas actividades dará lugar a determinados logros de ejecución (vía 5) resultando de ello el reforzamiento o la revisión de las percepciones de autoeficacia (vía 6).

Este modelo explicativo del desarrollo vocacional demuestra la complejidad de la teoría de la autoeficacia y su generalidad explicativa cuando se consideran los diversos componentes del pensamiento autorreferente así como sus interrelaciones.

Cabe destacar que si bien las expectativas de resultado ocupan un importante papel influyendo en los intereses, la contribución de las creencias de eficacia poseen mayor utilidad predictiva, ya que éstas en gran parte determinan las expectativas de resultado (vía 7). A continuación se representa el modelo propuesto:

Figura 2. Modelo de desarrollo de intereses a lo largo del tiempo. El modelo representa las influencias cognitivas y conductuales durante la niñez y la adolescencia (Lent, Brown \& Hackett , 1994)

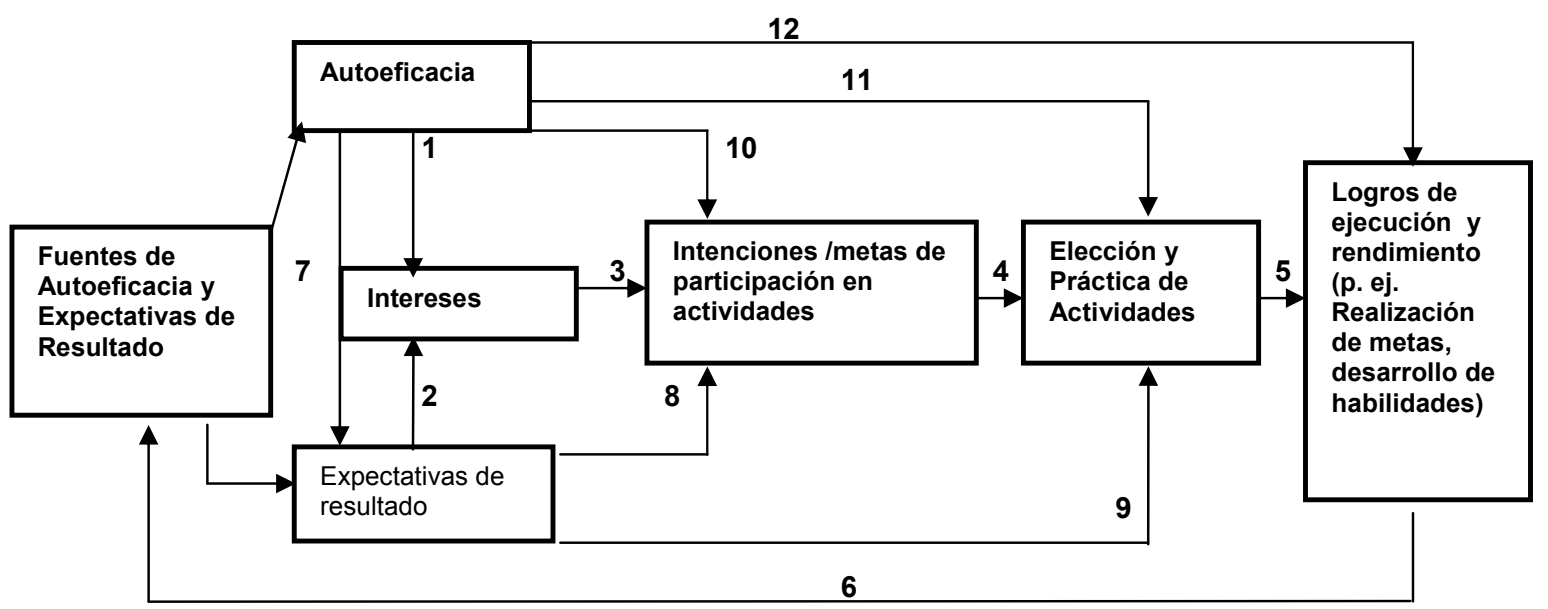

Los autores consideran que este proceso (representado en la figura 2) se repite continuamente a lo largo de la vida, siendo más fluido en los primeros años de vida ya que hacia la adolescencia tardía o la temprana adultez los intereses tienden a estabilizarse. Una vez que los intereses han cristalizado, serían necesarias experiencias de gran fuerza para provocar una revisión de los juicios de autoeficacia y de las expectativas de resultado, y de esta forma un cambio en los patrones básicos de intereses. Los autores sugieren a su vez, coincidiendo con Bandura (1987), que puede haber una demora temporal entre la adquisición de nuevas creencias de autoeficacia y 
la cristalización de intereses en una actividad que haya sido percibida previamente como neutral o aversiva por el individuo.

Bandura (Bandura \& Schunk, 1981), postula también que existe un efecto de umbral en la emergencia de intereses a partir de las creencias de autoeficacia. De esta forma, es necesario al menos un nivel moderado de autoeficacia para desarrollar y mantener interés en una actividad, pero un incremento adicional por encima del umbral puede no ocasionar incrementos lineales adicionales en los intereses. Por el contrario, niveles muy altos de autoeficacia en una determinada actividad pueden disminuir los intereses en ella, al quitarle su cualidad desafiante (Lent, Brown \& Hackett ,1994).

Como ya se señalo, las expectativas de resultados están parcialmente determinadas por las creencias de autoeficacia (vía 7), ya que aquellas personas que se consideran capaces en una actividad particular anticiparan resultados exitosos. Sin embargo las expectativas de resultados afectan a las metas tanto directa (vía 8) como indirectamente a través de los intereses (vías 2 y 3). Las expectativas de resultados realizan también una contribución independiente a la selección de actividades (vía 9).

Las creencias de autoeficacia afectan de forma directa a las metas y a las elecciones de actividades (vías 10 y 11), así como a los logros de ejecución y al rendimiento (debido a su rol mediacional entre las habilidades reales y el conocimiento del individuo, y su conducta posterior). Debido a su papel mas importante en la elección de actividades, como factor motivador, que en la determinación del rendimiento de la persona en las actividades elegidas, los autores no postulan vía alguna entre las expectativas de resultado y el rendimiento.

El efecto de las habilidades reales (incluidas dentro de las fuentes de autoeficacia) del individuo sobre los intereses es mediado por las creencias de autoeficacia, ya que las personas se basan más en las creencias acerca de sus habilidades que en sus habilidades reales (Lent, Brown \& Hackett, 1994). En numerosas investigaciones (Barak,1981) se han observado mayores correlaciones entre creencias de autoeficacia e intereses que entre habilidades medidas objetivamente (por medio de tests convencionales) e intereses, lo cual confirma el importante rol mediacional de la autoeficacia en la relación habilidad-intereses. 


\section{EI Modelo de elección de Carrera}

Básicamente, el modelo de elección de carrera propuesto incorpora la secuencia causal representada en el modelo de desarrollo de intereses vocacionales (véase figura 1). La diferencia fundamental es que en el presente modelo las Intenciones y metas de participación en actividades son representadas como metas de elección de carrera, y la variable Elección y Práctica de Actividades es representada específicamente como Acciones de elección de carrera. Tal como señalan los autores (Lent, Brown \& Hackett, 1994), este modelo puede ser visto como una extensión evolutiva del proceso de formación de intereses. Como puede verse, los autores dividen conceptualmente la fase de elección de carrera en tres etapas o componentes: a) la expresión de una meta de elección de alguna de las carreras de mayor interés para el sujeto, b) las acciones destinadas a la puesta en práctica de la elección (tales como inscribirse en una carrera determinada), y c) los logros de ejecución y el rendimiento subsecuente (los fracasos académicos, la aprobación y el éxito académico, por ejemplo), que crea un bucle de retroalimentación (Vía 6) afectando y dando forma al comportamiento vocacional futuro. El modelo propuesto por los autores para la explicación del proceso de elección de carrera es ilustrado en la figura 3. 
Figura 3. Modelo de selección de carrera. El modelo representa los factores personales, contextuales y experienciales que afectan al comportamiento de selección de carrera (Lent, Brown \& Hackett , 1994). Los factores que actúan como moderadores de relación se representan con línea punteada.

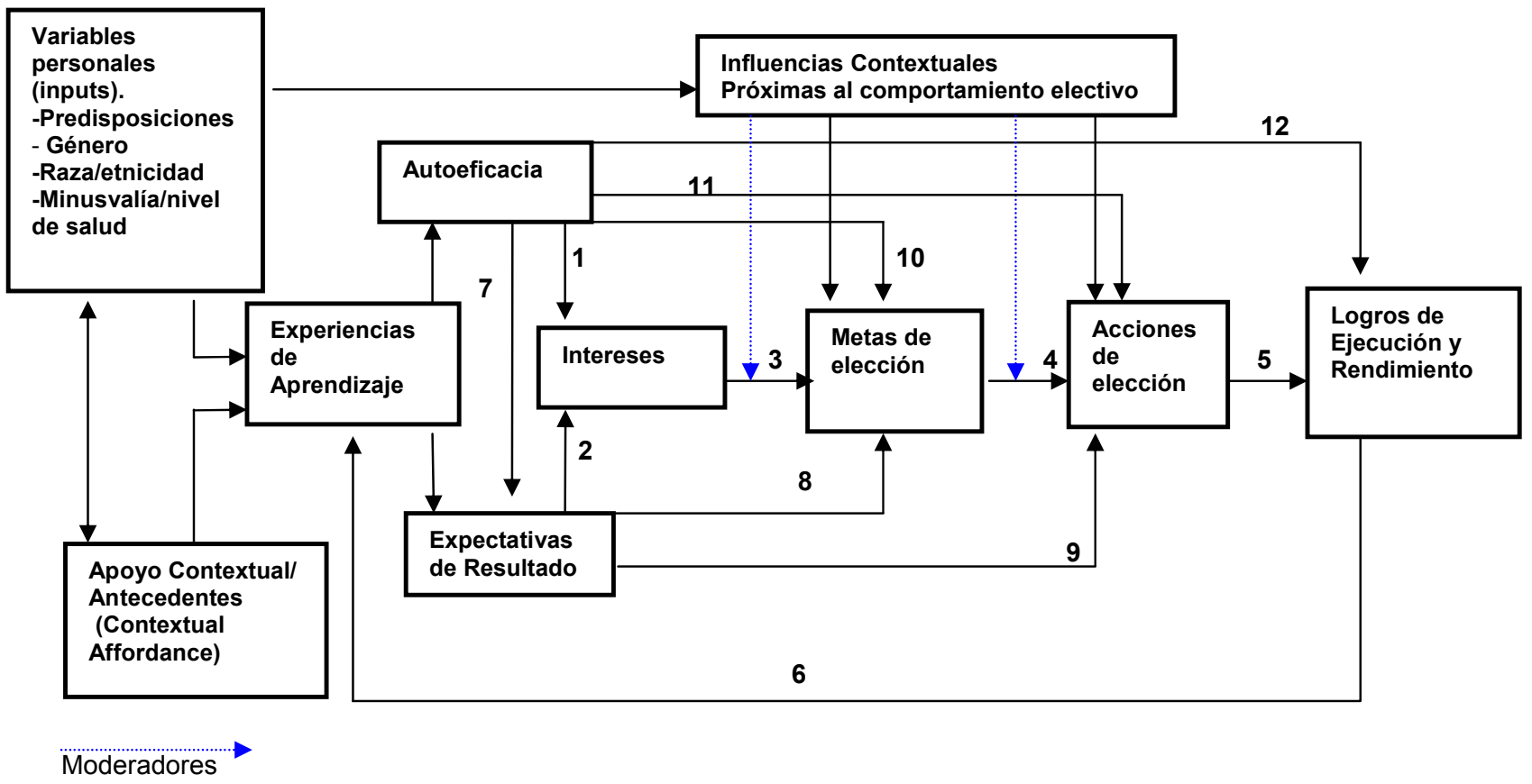

En el modelo, las elecciones no representan actos estáticos, sino que, por el contrario, son modificadas constantemente por los resultados del comportamiento del sujeto. De esta manera, si un sujeto elige una determinada carrera en la cual tiene muchas dificultades para cumplir con los requisitos del cursado, éste se vera forzado a una revisión inmediata de sus capacidades percibidas, conduciéndolo a un cambio en sus metas (elección de una nueva carrera, por ejemplo).

Subdividir conceptualmente el proceso de elección de carrera de esta forma permite resaltar el rol mediacional de las metas personales en el proceso de elección de carrera. Coincidiendo con la visión propuesta por Bandura (1987) del hombre como productor de su propio futuro y no como un mero predictor, los autores consideran que nuestras acciones de elección no son implantadas automáticamente por la presión del medio o determinadas directamente por nuestra historia personal. Por el contrario, la capacidad de autorregularnos por medio de metas, las cuales surgen del interjuego de la autoeficacia, las expectativas de resultados y los intereses, aporta una medida de agencia personal en la determinación de nuestro propio futuro vocacional, más allá de 
que las metas sean sensitivas a influencias involuntarias (Lent, Brown \& Hackett, 1994).

Los autores coinciden con la hipótesis fundamental de Hackett y Betz (1981), al señalar que la autoeficacia influiría en las elecciones de carrera, pero aportan un análisis mas detallado de las vías a través de las cuales actúa la autoeficacia y la forma mediante la cual ésta interactúa con otros mecanismos causales.

Como puede observarse en la figura 2, la autoeficacia y las expectativas de resultados actúan como codeterminantes de los intereses vocacionales (vía 1 y 2). Los intereses, a su vez, promueven metas de elección vocacional (intenciones o aspiraciones a comprometerse en una dirección vocacional particular) (vía3), las cuales aumentan la probabilidad de una acción de elección determinada (vía 4). Estas acciones de elección conducen al sujeto a dominios de rendimiento particulares y experiencias de logro que pueden alimentar o debilitar la autoeficacia y las expectativas de resultados, al servir como experiencias de aprendizaje (vía 6), afectando, de esta forma, la persistencia en la selección realizada. Las expectativas de resultados afectan a las metas de elección y a las acciones de selección tanto indirectamente a través de los intereses (vía 2), como directamente (vía 8 y 9 respectivamente). Cuanto más valorados sean los resultados percibidos, mayores probabilidades habrá de que un sujeto adopte metas vocacionales y cursos de acción determinados. La autoeficacia también es vista como afectando el proceso de elección a través de diversas vías: indirectamente, a través de las expectativas de resultado (vía 7) y de los intereses (vía 1), y directamente por medio de las metas de elección vocacional (vía 10), las acciones de elección (vía 11), y los logros de ejecución y el rendimiento subsecuente (vía 12).

Las metas poseen un número de propiedades que afectan su influencia en el comportamiento, tales como su intensidad y su firmeza. Por esto, se espera que aquellas metas firmemente sostenidas (lo cual indica seguridad en la elección, decisión y compromiso) promoverán mas acciones de selección que aquellas metas más débiles o menos firmemente sostenidas (Lent, Brown \& Hackett, 1994).

Los autores añaden que las metas tendrán un mayor efecto motivador sobre el comportamiento cuanto más claras y específicas sean, cuando sean percibidas como 
desafiantes, próximas en el tiempo al comportamiento, y relacionadas a conductas susceptibles de control voluntario (Lent, Brown \& Hackett , 1994).

\section{EI Modelo de Rendimiento}

En el modelo propuesto por Lent, Brown \& Hackett (1994), se define al rendimiento en un sentido amplio, incluyendo niveles de logro (notas en las materias, por ejemplo) así como índices de persistencia conductual (estabilidad en la carrera elegida). Este modelo, incluido en la figura 2 , es de utilidad en la explicación de los logros de ejecución relacionados a las metas personalmente seleccionadas, o, en el caso de actividades que son impuestas o demandadas por agentes externos, personalmente adoptadas.

Los logros de ejecución y el rendimiento se ven afectados, en parte, por nuestras metas, que colaboran en la autorregulación del comportamiento, movilizando y sosteniendo acciones relevantes para la ejecución de una tarea (vías 4 y 5). Coincidiendo con Bandura (1987), los autores consideran que la autoeficacia ejerce tanto un efecto directo en el rendimiento (ya que las creencias de autoeficacia organizan y dirigen las habilidades reales del sujeto; vía 12) como indirecto, a través de las metas y las acciones de elección. El efecto de las expectativas de resultado es visto como mediado por las metas y las acciones de selección (vías 8 y 9). Como se señalo anteriormente, los autores señalan una vía de retroalimentación (vía 6) entre los logros de ejecución y el rendimiento, y posteriores elecciones y comportamientos. Un rendimiento exitoso favorecerá percepciones de autoeficacia y expectativas de resultados positivas, fortaleciendo de esta forma nuestros intereses y nuestras metas.

Las metas de elección cumplen un rol de importancia en el dominio de rendimiento elegido (tipo de tareas académicas o laborales elegidas), pero las acciones de elección no determinan per se el nivel de rendimiento logrado. La calidad del rendimiento logrado va a depender, en gran parte de las metas de rendimiento que el sujeto se proponga (Lent, Brown \& Hackett, 1994). Un sujeto cuya meta de elección sea estudiar una carrera determinada, también se fijará metas relacionadas al nivel de rendimiento que se propone lograr en las materias requeridas para el cursado de la misma. 
La metas de rendimiento son vistas como cumpliendo un rol de gran importancia ayudando a la persona a regular el gasto de energía, promover la persistencia en las tareas elegidas, y al dirigir la atención de las personas hacia su propia conducta y los resultados de la misma. Es por ello que los autores proponen una serie de relaciones específicas entre la autoeficacia, las expectativas de resultado, las metas y submetas de rendimiento y el nivel de rendimiento logrado.

La teoría social cognitiva distingue entre metas distantes y submetas más próximas. Las metas finales influyen en la elección de los cursos de acción pero están demasiado alejadas en el tiempo como para funcionar como incentivos efectivos para la acción presente. Las submetas próximas movilizan en forma efectiva el esfuerzo en las actividades que el sujeto realiza en el presente y conducen a aspirar a las metas últimas, creando las condiciones más favorables para la automatización (Bandura, 1983). Las relaciones propuestas son representadas en la figura 4.

Figura 4: Modelo de rendimiento. Se representan los roles de la Habilidad real del individuo, la Autoeficacia, las Expectativas de Resultados y las Metas de Rendimiento (Lent, Brown \& Hackett, 1994).

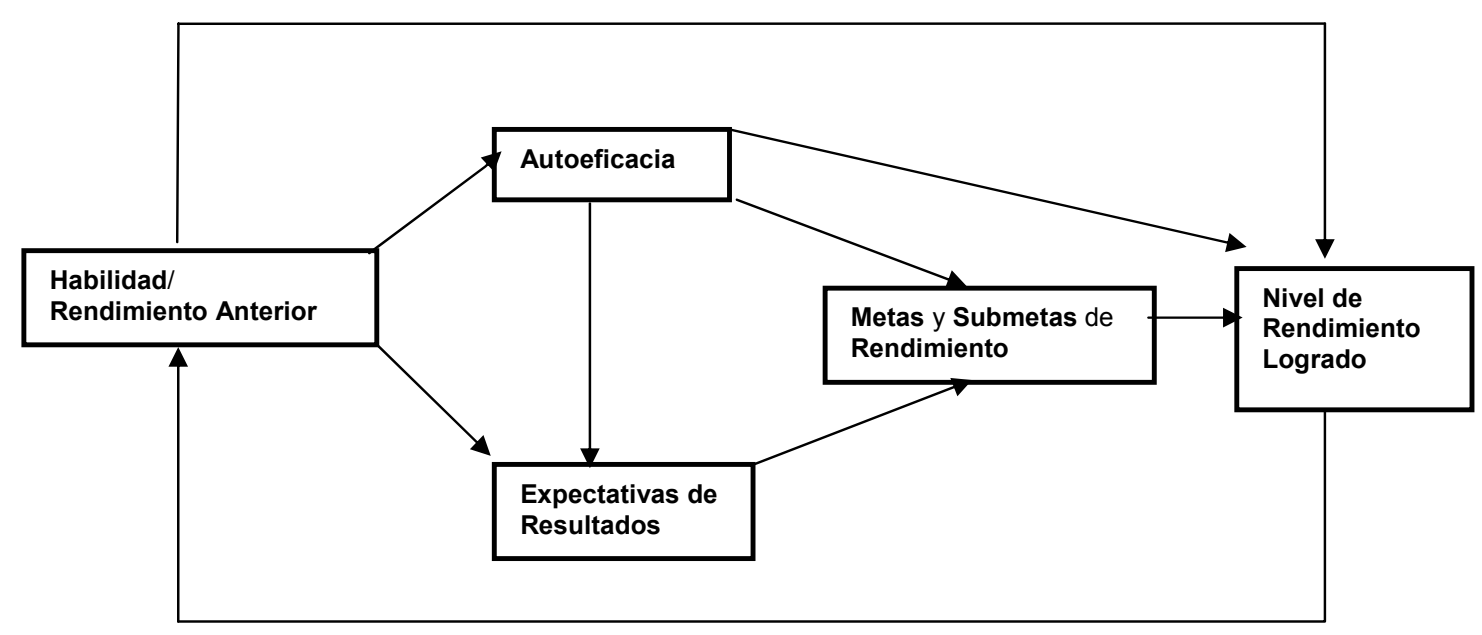

En la figura 4, puede observarse que las habilidades reales del individuo (evaluadas por medio de índices de rendimiento anterior, logros anteriores o aptitudes) afectan el nivel de rendimiento posterior a través de dos vías: a) directamente, en forma de habilidades desarrolladas de maestría en las tareas y b) indirectamente, a través de los juicios de autoeficacia y las expectativas de resultados. La ejecución correcta de tareas de complejidad requiere, sumado a un establecimiento de metas de 
rendimiento adecuadas, de habilidades de maestría en las tareas. Por ejemplo, para obtener buenas notas en la carrera de músico, el estudiante necesitará poseer habilidades en el manejo de la armonía, la lectura musical, la apreciación melódica y la composición musical, entre otras habilidades, además de fijarse metas de rendimiento superior. La relación de las aptitudes o habilidades reales del individuo y las creencias de autoeficacia a menudo no son tan simples como "a mayores habilidades mayor autoeficacia", sino que por el contrario a menudo nos encontramos con individuos que sobrestiman o subestiman su potencial "real" (reflejado en sus notas o en los puntajes de tests objetivos). Tales disparidades pueden resultar de una variedad de experiencias o influencias cognitivas (Lent, Brown \& Hackett, 1994). Una autoeficacia altamente sobrestimada puede ser contraproducente, ya que enfrenta al individuo a mayores posibilidades de fracaso; creencias pesimistas de autoeficacia pueden llevar al individuo a evitar actividades para las cuales es altamente capaz limitando sus experiencias de aprendizaje y afectando su desarrollo académico y de carrera. La Teoría Social Cognitiva asume que el nivel óptimo en las creencias de autoeficacia es aquel que excede mínimamente al nivel de habilidad real del individuo, ya que alienta al individuo a enfrentar actividades desafiantes que favorecen el desarrollo de la autoeficacia, ayudándolo a perseverar frente a las dificultades y a tolerar las frustraciones que puedan presentársele en el transcurso de la actividad (Bandura, 1987).

Como puede observarse, en el modelo se omite a los intereses como una variable mediacional ya que los autores ven a los intereses vocacionales como un mediador de más importancia para la elección de actividades académicas y de carrera que para la selección de metas de rendimiento. Numerosas investigaciones han destacado el mayor valor predictivo de los intereses para la elección de carrera que para la predicción de criterios relacionados al rendimiento (Hansen,1984). Como puede verse en la figura 3, la autoeficacia afecta al nivel de rendimiento tanto directamente como indirectamente, a través de las metas y submetas de rendimiento. Además el modelo incorpora el rol de las expectativas de resultados complementando a la autoeficacia, y añade una vía que relaciona el nivel de rendimiento logrado con la autoeficacia y las expectativas de resultados subsecuentes. El rol de las expectativas de resultados como factor motivador independiente se hace evidente en aquellas 
actividades en las cuales los resultados están débilmente relacionados con la calidad del rendimiento (una persona que evita elegir una carrera determinada para la cual se siente altamente capaz debido a que anticipa resultados negativos de esa elección, como puede ser el poco apoyo de su familia o amigos, podría ser un claro ejemplo).

Bandura (1987) ha propuesto una variedad de factores que pueden moderar la relación entre autoeficacia y el rendimiento posterior tales como: la existencia de incentivos apropiados para llevar a cabo la acción, evaluaciones de autoeficacia bajo condiciones que favorecen la honestidad, evaluación de autoeficacia y rendimiento realizadas en proximidad temporal y teniendo en cuenta dimensiones comunes, metas de rendimiento claras, conocimiento preciso de las propias capacidades en las personas evaluadas, entre otros.

\section{Variables contextuales, personales y experienciales.}

Lent, Brown \& Hackett (1994) señalan que aún cuando los modelos anteriormente presentados parecen representar un conjunto de procesos normativos que tienen lugar en condiciones de óptimo control voluntario, es importante reconocer que en el mundo real existen una serie de importantes factores adicionales (económicos, culturales y personales) que moderan el poder explicativo de los modelos. Estos factores actúan como variables intervinientes, fortaleciendo o debilitando las relaciones entre intereses, metas y acciones. Es por ello que los autores proponen algunas variables adicionales que actúan a modo de inputs, tales como algunas variables personales (atributos físicos, por ej.); características del medio físico, social y cultural; y experiencias de aprendizaje relevantes para él comportamiento vocacional. Como puede verse en la figura 2 , se proponen tres vías causales predominantes a través de las cuales los factores personales, contextuales y experienciales pueden influir a los intereses vocacionales y en el comportamiento relacionado a la selección de carreras. Estos factores pueden actuar como: a) precursores de variables sociocognitivas, b) moderadores de algunas de las relaciones propuestas y c) facilitadores o disuasores directos (practicas discriminatorias que restringen el acceso a ciertas opciones de carrera, por ej.). Algunos factores pueden operar a través de más de un modo de influencia.

Como ya se señaló, las creencias de autoeficacia están fuertemente determinadas y son modificadas de acuerdo a cuatro fuentes experienciales: logros de

Evaluar.

2003, $n^{\circ} 3$ (julio) 
ejecución, experiencia vicaria, persuasión verbal y estado fisiológico. Similares fuentes experienciales pueden afectar a las expectativas de resultados. De esta manera, las personas anticipan determinados resultados extrínsecos e intrínsecos (autoevaluativos) de sus acciones al recordar los resultados que las acciones propias produjeron en el pasado (estudiar trae como consecuencia buenas notas y autoaprobación, por ej.), observando las consecuencias experimentadas por otros (modelado) y atendiendo a los relatos de otra persona acerca de las contingencias de reforzamiento. Además, Bandura (1997) señala que las expectativas de resultados están parcialmente determinadas por las creencias de autoeficacia en aquellas situaciones donde los resultados de las acciones dependen en gran medida de la calidad de la conducta y del buen rendimiento.

Entre los diferentes factores personales que interactúan con la autoeficacia, las expectativas de resultados y las metas, los autores se centran particularmente en dos de ellos, los cuales solo han sido tratados en forma superficial por la mayoría de las teorías: el género y la raza / etnicidad. En el modelo propuesto, los efectos del género y la etnicidad, sobre los intereses, las elecciones y el rendimiento serán parcialmente mediados por experiencias diferenciales de aprendizaje determinadas por estos factores, las cuales afectarán directamente a las creencias de autoeficacia y a las expectativas de resultados. Los factores culturales y de género determinan, a su vez, la estructura de oportunidades a lo largo de la cual son formadas e implementadas las metas vocacionales.

Finalmente, se incluyen también atributos heredados como variables personales. En el marco propuesto se considera que existen ciertas potencialidades básicas, las cuales, en interacción con los recursos del medio, pueden explicar los determinantes genéticos de los intereses y de las otras variables sociocognitivas consideradas. Lent, Brown \& Hackett (1994) consideran que el efecto de la determinación genética de los intereses opera, esencialmente, por medio de ciertas experiencias de aprendizaje que dan forma al substrato heredado transformándolo en habilidades relevantes para el comportamiento vocacional, las cuales, a su vez, son la fuente de las creencias de autoeficacia y de las expectativas de resultado. La susceptibilidad diferencial producto de la dotación genética así como la práctica de actividades y la exposición selectiva al reforzamiento son factores codeterminantes de 
creencias diferenciales de autoeficacia y de expectativas de resultado, las cuales promueven particulares patrones de intereses.

Coincidiendo con diferentes autores (Astin, 1984; Hansen, 1984) se considera que el desarrollo del comportamiento vocacional se ve influido por una serie de factores integrados en forma de una "estructura de oportunidades". Esta estructura de oportunidades tiene como una de sus funciones el acrecentar o disminuir el control voluntario en el proceso de elección de carrera. Muchos de los modelos propuestos para explicar el comportamiento vocacional se centran en el ser humano como un agente libre de determinaciones en la elección de sus actividades, enfatizando las variables personales y dejando en segundo plano los determinantes contextuales. Por el contrario, el presente modelo pone un especial énfasis en aquellos factores del contexto que facilitan o restringen el control voluntario en el proceso de elección de carrera Sin embargo, tal como puede verse en la figura 2, los autores han dividido estos factores en dos subgrupos, basados en la relativa proximidad a los puntos de elección de carrera: a) influencias distales, que actúan como antecedentes que preceden y dan forma a los intereses y a las otras variables de autocognición (exposición diferencial a modelos de tareas y de roles, apoyo emocional y económico para iniciar determinadas actividades, procesos de socialización de roles culturales y de género, por ej.; véase apoyo contextual/antecedentes); b) influencias contextuales próximas al comportamiento electivo que actúan como factores determinantes críticos (red de contactos personales, barreras estructurales teles como practicas discriminatorias de selección laboral, por ej.).

Ambos conjuntos de influencias poseen elementos que se solapan y existen también algunos elementos, tales como el apoyo familiar, que están siempre presentes y que juegan un rol fundamental debido a su influencia constante en nuestro comportamiento vocacional. También existen algunos eventos que ejercen un potente efecto directo en la formación de nuestros intereses y elecciones, y en su implementación (tales como la discriminación en el empleo). Simplificando, en el modelo propuesto los factores contextuales ayudan a dar forma a las experiencias de aprendizaje, que son la fuente de los intereses y selecciones personales, y constituyen la estructura de oportunidades real y percibida dentro de la cual los planes de carrera son diseñados e implementados.

Evaluar.

2003, n $^{\circ} 3$ (julio) 
Olaz, $F$.

Algunos factores próximos al comportamiento electivo y que constituyen esta estructura de oportunidades actúan como moderadores que afectan algunas de las vías causales propuestas. Estos factores moderan la relación de a) intereses con metas de elección y b) metas con acciones. De esta manera el modelo propuesto sugiere que las relaciones intereses-metas y metas-acciones van a tender a ser más fuertes entre aquellas personas que perciben mayores condiciones beneficiosas de parte del entorno y más débiles entre aquellas personas que perciben condiciones menos favorables.

Además de este importante rol de moderadores, existen algunos factores del medio que ejercen un potente efecto directo en el proceso de elección de carrera influyendo directamente en el proceso o afectando la fortaleza relativa de algunos determinantes cognitivos. Es así como, por ejemplo, la necesidad económica puede llevar a las personas hacia determinadas carreras basando su elección en la disponibilidad de trabajo, su autoeficacia y sus expectativas de resultado que en sus intereses vocacionales (vías 9 y 11, figura 2). Por ejemplo, una persona con fuertes intereses por una carrera artística puede elegir una carrera como Administración de Empresas debido a que se considera capaz de desempeñarse en forma exitosa en ella y por considerar que la remuneración monetaria percibida por su ejercicio será mucho mayor que la que podría recibir como pintor, músico o poeta.

\section{Discusión}

La elaboración de modelos explicativos del desarrollo del comportamiento vocacional de creciente complejidad, basados ya no solamente en consideraciones teóricas sino en los resultados de numerosas investigaciones empíricas y que incorporan numerosas variables, están permitiendo una mayor comprensión del proceso de elección de carreras así como de las complejas relaciones entre las variables frecuentemente asociadas a tal proceso.

De los numerosos modelos propuestos se destaca principalmente el propuesto por Lent, Brown \& Hackett (1994), como un esfuerzo de integración y unificación teórica entre constructos aparentemente diversos, diseñados para explicar resultados comunes, así como por su énfasis en la investigación empírica de las hipótesis propuestas por ellos. El modelo es un intento de extensión de la línea iniciada por 
Hackett \& Betz, incorporando aspectos de la teoría Social Cognitiva y permite un análisis más complejo y refinado del comportamiento vocacional, ampliando los aportes que el constructo de la Autoeficacia puede realizar por sí solo.

\section{Referencias}

Astin, H.S. (1984) Attitudes, personality, and behavior. Stony Stratford. United Kingdom:Open University Press.

Bandura, A.; \& Schunk, D.H. (1981) Cultivating competence, self efficacy, and intrisic interest through proximal self-motivation. Journal of Personality and Social Psychology, 41, 586-598.

Bandura, A. (1987) Pensamiento y Acción. Barcelona: Martínez Roca.

Bandura, A. (1997). Self-efficacy: The exercise of control. New York: Freeman.

Bandura, A. (1999). Auto-eficacia: Cómo afrontamos los cambios de la sociedad actual. Biblioteca de Psicología. Bilbao, Spain: Desclée De Bower

Bandura, A.(1983) Modelo de Causalidad en la Teoría del Aprendizaje Social. En Mahoney,M \& Freeman, A. comp.(1988).Cognición y Psicoterapia. Buenos Aires: Paidós.

Barak, A. (1981) Vocational Interests: A cognitive view. Journal of Vocational Behavior, 19, 1-14.

Gómez Benito, J. (1987). Meta-análisis. Barcelona: P.P.V

Hackett, G. \& Betz, N. (1981) A self-efficacy approach to the career development of women. Journal of Vocational Behavior. 18, 326-339.

Hansen, J. C. (1984). Response to the meaning of work in women's lives. The Counseling Psichologist, 12, 147-149.

Kerlinger, F. \& Lee, H. (2002) Investigación del Comportamiento. (Cuarta edición).México: Mc Graw Hill.

Krumboltz, J.D. (1996) A Learning Theory of Career Counseling. En Savickas, M \& Walsh B. W. (Eds.), Handbook of Career Counseling Theory and Pratice. Palo Alto, CA: Davies- Black Publishing.

Evaluar.

2003, $n^{\circ} 3$ (julio) 
Lent, R.; Brown, S.; \& Larkin, K (1984) Relation of self efficacy expectations to academic achievement and persistence. Journal of Counseling Psychology. 31, 356-362.

Lent, R.; López, F.;Bieschke,K.(1991) Mathematics self-efficacy: Sources and relation to science-based career choice. Journal of Counseling Psychology. 38, 424-430.

Lent, R.; López, F.;Bieschke,K.(1993) Predicting Mathematics-Related Choice and Succes Behaviors: Test of an Expanded Social Cognitive Model. Journal of Vocational Behavior. 42, 223-236.

Lent, R.; Brown, D; Hackett,G. (1994) Toward a Unifying Social Cognitive Theory of Career and Academic Interest, Choice, and Performance. Journal of Vocational Behavior.45, 79-122.

Pajares, F. (1997). Currents directions in self-efficacy research. En Maehr,M. \& Pintrich, P. (Eds.) Advances in motivation and achievement. 1-49. Greenwich, CT: JAI Press.

Schunk, D.H. (1989) Self-efficacy and Achievement Behaviors. Educational Psychology Review. 1, n 3. 173-207 